УДК $340.15 ; 347.157 ; 343.224$

\title{
INDEX ROMANORUM VERBORUM IURIDICORUM AD AETATEM PERTINENTIUM (INDEX OF ROMAN LEGAL SOURCES ASSOCIATED WITH AGE)
}

\author{
W. Kosior \\ University of Rzeszow, \\ ul. Grunwaldzka 13, Rzeszów, Poland, 35-068, \\ e-mail:wojciech.kosior@gmail.com
}

The main goal of the studies conducted by the Author was to collect and describe all age categories and age limits appearing in Roman law. The research was conducted with accordance to the historical periodization of Roman law - from the archaic period till the Justinian era. This elaboration presents the essence of the scientific research devoted to age categories in Roman law. The article was elaborated in a table-article manner to be more clear. It contains all age categories and numeric age limits which were mentioned in ancient Roman juridical sources. It is the first work of such type in the history of Roman law research. The presented Index of Roman Legal Terms Associated with Age (Index Romanorum Verborum luridicorum Ad Aetatem Pertinentium) is a comprehensive catalogue of legal sources ranging from archaic law to Justinian law, which include age-related terms. The following Index would certainly serve all Roman law researchers and other people interested in the ancient world.

Keywords: Roman law, age, infantia, impuberes, puberes.

DOI: http://dx.doi.org/10.30970/vla.2018.67.148

In every legal system, regardless of whether we mean antique or modern law, there have always been and there are regulations that have specific effects depending on the age of a given person. Such regulations are known in all contemporary legal systems of the world. What is more, such norms are currently present in every branch of law. For example, in civil law, age serves to determine the legal capacity; in criminal law - the criminal liability depends on the age of the perpetrator; in constitutional and administrative law, age designates active and passive electoral rights or sets census to perform specific professions, for example a president or a member of parliament.

There were also such norms in Roman law. In the subject literature, age categories appear primarily when discussing legal capacity. As a rule, the following age categories were distinguished: children (infantia) 0-7 years of age, immature ones (impubertas) 7-14 years of age (or 12 years for women), mature people under 25 years of age (puberes minores $X X V$ annis) and adults over 25 years of age (puberes maiores $X X V$ annis). In addition, the law of ancient Romans also provided for a number of other regulations where certain rights or obligations were associated with the achievement of a specific age.

The main area of my scientific interest is the role of age in Roman law. Being more specific, with my research I am trying to answer to question how and in what manner human's age influenced legal position of a person in Roman law. Such research has not been conducted before. To achieve my scientific goal, there has been a necessity to catalogue the complete database of Roman law legal sources in which the age was mentioned. It was the first step to find justifications of many hypotheses, which I set in my research. It is not only about answering the question whether the Romans knew other age categories and age limits than the above-mentioned ones, but I am mainly interested

(C) Kosior W., 2018 
in presenting what the achievement of a given age was based on legal grounds. What did it mean for the Romans to exceed the exact age in the sphere of their rights and obligations.

In this elaboration, I am presenting the essence of my academic research, i.e. the database in which all Roman juridical sources from the archaic period till the Justinian era, which contain age categories or limits, are catalogued. This Index is the first work of such type in the history of Roman law research. The performance of this assumption would certainly serve all Roman law researchers and other people interested in the ancient world. Thanks to this, anyone interested in any matter concerning age in Roman law or age limit will be able to use the index to find the legal text in which the category or age occurs.

The presented Index consists of two parts: (1) Age limits in Roman law that is the catalogue of all numerical limits, which were mentioned in legal texts and (2) Age categories in Roman law, which is the database of human life's legal phases determined by the exact numeric age limits. To present this Index in the most intelligible and readable manner, I decided to demonstrate it in the table format.

To elaborate the following Index I employed all preserved collections of Roman law juridical texts. In the table the proper abbreviations were used. Here is the list of the sources and their short forms: Leges Regiae (L. Reg.); Lex XII Tabularum (L. Duodecim); Tabula Heracleensis (TH.); Gaii Institutiones (G.); Gaius Augustodunensis (G. Aug.); Sententiae Pauli (Paul.); Pauli Sententiarum Interpretatio (Interpr. Paul. sent.); Fragmenta Vaticana (Vat. fr.); Collatio legum Mosaicarum et Romanarum (Coll.); Epitome Ulpiani (Epit. Ulp.); Epitome Gai (Epit. Gai.); Codex Theodosianus (CTh.); Theodosiani Novellae (Nov. Theodos./ Nov. Mai./ Nov. Val.); Iustiniani Institutiones (I.); Iustiniani Digesta (D.); Codex Iustinianus (C.); Iustiniani Novellae (Nov. Iust.).

\section{Age limits in Roman law}

\begin{tabular}{|c|c|c|c|c|c|c|}
\hline $\begin{array}{l}3 \\
\text { months }\end{array}$ & D. $25,4,1,10$ & $\begin{array}{l}\text { Edict. Salv. 2, } \\
21,118\end{array}$ & & & & \\
\hline $\begin{array}{l}6 \\
\text { months }\end{array}$ & D. $25,4,1,10$ & $\begin{array}{l}\text { Edict. Salv. 2, } \\
21,118\end{array}$ & & & & \\
\hline 1 year & $\begin{array}{l}\text { G. } 1,29 \\
\text { G. } 1,32 \\
\text { G. } 1,32 \mathrm{a}\end{array}$ & $\begin{array}{l}\text { G. } 1,73 \\
\text { Epit. Ulp. } 3,3 \\
\text { Vat. fr. } 321\end{array}$ & $\begin{array}{l}\text { D. } 1,6,6 \\
\text { D. } 6,2,12,5 \\
\text { D. } 9,2,23,7\end{array}$ & $\begin{array}{l}\text { D. } 23,4,26 \\
\text { D. } 25,4,1,10\end{array}$ & $\begin{array}{l}\text { D. } 28,6,45 \text {. } \\
\text { D. } 32,60\end{array}$ & $\begin{array}{l}\text { D. } 50,16, \\
132 \\
\text { D. } 50,16, \\
134\end{array}$ \\
\hline 3 years & L. Reg, 2, 10 & L. Reg, 1, 4 & $\begin{array}{l}\text { Edict. Salv. } \\
2,21,118\end{array}$ & Vat. fr. 321. & & \\
\hline 6 years & Paul. 1, 21, 13 & CTh. $8,18,4$ & & & & \\
\hline 7 years & $\begin{array}{l}\text { CTh. } 8,18,8 \\
\text { CTh. } 12,1,19\end{array}$ & D. $23,1,14$ & D. $26,7,1,2$ & D. $32,41,4$ & C. $6,30,18$ & $\begin{array}{l}\text { C. } 6,30, \\
18,4\end{array}$ \\
\hline 8 years & CTh. 12, 1, 19 & D. $28,6,43,1$ & & & & \\
\hline $\begin{array}{l}10 \\
\text { years }\end{array}$ & $\begin{array}{l}\text { L. Reg, } 2,10 \\
\text { Vat. fr. } 321\end{array}$ & $\begin{array}{l}\text { CTh. } 3,5,11 \\
\text { CTh. } 3,5,11,1\end{array}$ & $\begin{array}{l}\text { CTh. } 3,5 \text {, } \\
\text { 11, Interpr. } \\
\text { D. } 28,6,21\end{array}$ & $\begin{array}{l}\text { D. } 28,6,38,1 \\
\text { D. } 28,6,43,1\end{array}$ & C. $6,43,31$ & C. $7,7,1,5$ \\
\hline 11 & CTh. $3,5,11,1$ & CTh. $3,5,11$, & & & & \\
\hline
\end{tabular}




\begin{tabular}{|c|c|c|c|c|c|c|}
\hline \multicolumn{2}{|l|}{ years } & \multicolumn{5}{|l|}{ Interpr. } \\
\hline 12 & G. 2,112 & CTh. $3,5,11,1$ & D. $23,1,9$ & D. $25,7,1,4$ & D. $27,6,11,4$ & D. 48,5 , \\
\hline years & G. 2,113 & CTh. $3,5,11,3$ & D. $23,2,62$, & D. $26,5,13,2$ & D. $28,1,5$ & 14,8 \\
\hline & Paul. 3, 4a, 1 & CTh. $3,5,11$, & 2 & D. $26,5,26$ & D. $28,6,2$ & D. 50,15 , \\
\hline & Epit. Ulp. 16, 1a & Interpr. & D. $23,2,4$ & D. $27,6,11$ & D. $33,1,21,5$ & 3 \\
\hline & Epit. Ulp. 20, 15 & I. 1,22 & D. $23,3,74$ & D. $27,6,11,3$ & D. $42,5,17,1$ & C. $5,4,24$ \\
\hline & Epit. Gai.2, 2, 2 & I. $2,16,1-9$ & $\begin{array}{l}\text { D. } 24,1,31 \text {, } \\
27\end{array}$ & & & C. $5,60,3$ \\
\hline
\end{tabular}

13 CTh. 4, 14, 1,

years Interpr.

\begin{tabular}{|c|c|c|c|c|c|c|}
\hline $\begin{array}{l}14 \\
\text { years }\end{array}$ & $\begin{array}{l}\text { G. 1, 40 } \\
\text { G. 1, 196 } \\
\text { G. 2, 113 } \\
\text { Paul. 3, 4a, 1 } \\
\text { Epit. Ulp. 11, } 28 \\
\text { Epit. Ulp. 16, 1a }\end{array}$ & $\begin{array}{l}\text { Epit. Gai.1, 1, } \\
7 \\
\text { Epit. Gai.2, 2, } \\
2 \\
\text { CTh. } 4,8,6,3 \\
\text { CTh. } 4,8,6 \text {, } \\
\text { Interpr. } \\
\text { Nov. Theodos. } \\
\text { 11, Interpr. } \\
\text { I. } 1,6,7\end{array}$ & $\begin{array}{l}\text { I. } 1,22 \\
\text { I. } 2,16,1 \text { - } 9 \\
\text { D. } 27,6,11 \\
\text { D. } 28,1,5 \\
\text { D. } 28,6,2 \\
\text { D. } 28,6,2,5\end{array}$ & $\begin{array}{l}\text { D. } 28,6,7 \\
\text { D. } 28,6,15 \\
\text { D. } 28,6,21 \\
\text { D. } 28,6,33 \\
\text { D. } 28,6,381 \\
\text { D. } 28,6,43,1\end{array}$ & $\begin{array}{l}\text { D. } 31,69,2 \\
\text { D. } 33,1,21,5 \\
\text { D. } 34,1,14 \\
\text { D. } 34,3,28 \\
\text { D. } 48,18,10 \\
\text { D. } 48,18,15 \text {, } \\
1\end{array}$ & $\begin{array}{l}\text { D. } 50,15, \\
3 \\
\text { C. } 5,4,24 \\
\text { C. } 5,60,3 \\
\text { C. } 6,22,4 \\
\text { C. } 6,58,4 \\
\text { C. } 7,64,2\end{array}$ \\
\hline $\begin{array}{l}15 \\
\text { years }\end{array}$ & $\begin{array}{l}\text { CTh. } 4,14,1 \text {, } \\
\text { Interpr. }\end{array}$ & $\begin{array}{l}\text { CTh. } 4,3,1 \text {, } \\
\text { Interpr. }\end{array}$ & $\begin{array}{l}\text { D. } 10,2,39 \text {, } \\
2\end{array}$ & D. $35,1,36,1$ & $\begin{array}{l}\text { Nov. Iust. } \\
100,2\end{array}$ & \\
\hline $\begin{array}{l}16 \\
\text { years }\end{array}$ & D. $35,1,36,1$ & D. $50,5,2$ & CTh. $6,4,1$ & & & \\
\hline $\begin{array}{l}17 \\
\text { years }\end{array}$ & $\begin{array}{l}\text { Edict. Salv. } \\
1,6,14 \text {. }\end{array}$ & I. $1,6,5$ & I. $1,6,7$ & D. $3,1,1,3$ & & \\
\hline $\begin{array}{l}18 \\
\text { years }\end{array}$ & $\begin{array}{l}\text { Paul. 3, 4a, } 2 \\
\text { CTh. 2, 17, 1, } 1 \\
\text { CTh. 2, 17, 1, } \\
\text { Interpr. } \\
\text { CTh. 12, 1, } 7\end{array}$ & $\begin{array}{l}\text { CTh. 12, 1, } 19 \\
\text { CTh. 12, 1, 19, } \\
\text { Interpr. } \\
\text { CTh. 12, 1, 58, } \\
2\end{array}$ & $\begin{array}{l}\text { CTh, 13, 4, } \\
1 \\
\text { I. } 1,6,7 \\
\text { I. } 1,11,4\end{array}$ & $\begin{array}{l}\text { D. } 1,7,40,1 \\
\text { D. } 33,2,37 \\
\text { D. } 34,1,14\end{array}$ & $\begin{array}{l}\text { D. } 35,1,101 \text {, } \\
2 \\
\text { D. } 40,2,13 \\
\text { D. } 42,1,57\end{array}$ & $\begin{array}{l}\text { C. } 2,44,2 \\
\text { Nov. Iust. } \\
115,3,13 \\
\text { Nov. Iust. } \\
123,13\end{array}$ \\
\hline
\end{tabular}

19 CTh. 7, 13,1

years

\begin{tabular}{|c|c|c|c|c|c|c|}
\hline 20 & G. 1,38 & CTh. $6,4,2$ & D. $1,10,2$ & D. $36,1,64,1$ & D. $40,2,16,1$ & D. 40,13 , \\
\hline & G. 1,40 & CTh. $7,22,2$ & D. $18,7,4$ & D. $40,1,1$ & D. $40,2,19$ & 1 \\
\hline & G. 1,41 & CTh. $13,10,4$ & D. $22,5,20$ & D. $40,1,4,8$ & D. $40,2,20,4$ & D. 40,13 , \\
\hline & Interpr. Paul. & CTh. $14,3,5$ & D. $27,2,3,5$ & D. $40,1,16$ & D. $40,4,3$ & 3 \\
\hline & sent. $5,2,3,4$ & CTh. $14,9,1$ & D. $28,3,6,5$ & D. $40,1,12$ & D. $40,4,5,18$ & D. 40,13 , \\
\hline & Vat. fr. $177 \mathrm{a}$ & Nov. Val. 35, & D. 30,102 & D. $40,2,2$ & D. $40,5,34,1$ & 4 \\
\hline & Epit. Ulp. 1, 13 & 10 & D. $33,7,3,1$ & D. $40,2,4,2$ & D. $40,9,16$ & D. 45,1 , \\
\hline & Epit. Ulp. 16, 1 & I. $1,3,4$ & D. $34,1,15$ & D. $40,2,6$ & D. $40,12,7$ & 66 \\
\hline & Epit. Gai.1, 1, 7 & I. $1,6,4$ & D. $34,3,28$, & D. $40,2,11$ & D. $40,12,40$ & C. $2,30,2$ \\
\hline & CTh. $2,17,1$ & I. $1,6,7$ & 8 & D. $40,2,15$ & & C. $2,30,3$ \\
\hline & CTh. $2,17,1$, & D. $1,5,5,1$ & D. $35,1,113$ & & & C. $2,44,2$ \\
\hline & Interpr. & & & & & $\begin{array}{l}\text { C. } 7,10,6 \\
\text { C. } 7,16,16\end{array}$ \\
\hline
\end{tabular}

24 CTh. $7,20,4,3 \quad$ D. $26,7,56$

years 


\begin{tabular}{|c|c|c|c|c|c|c|}
\hline $\begin{array}{l}25 \\
\text { years }\end{array}$ & $\begin{array}{l}\text { G. 4, 57 } \\
\text { Edict. Salv. } \\
\text { 1,6,14 } \\
\text { Edict. Salv. } \\
\text { 1,10,44 } \\
\text { G. Aug. 26 } \\
\text { Paul. 1, 9, 1 } \\
\text { Paul. 1, 9, 4a } \\
\text { Paul. 1, 9, 5b } \\
\text { Paul. 1, 13a, 1e } \\
\text { Paul. 5, 12, 4a } \\
\text { Paul. 5, 12, 23 } \\
\text { Paul. 1,9, } \\
\text { Paul. 1, 9, 3 } \\
\text { Paul. 1,9, 4 } \\
\text { Paul. 1, 9, 4b } \\
\text { Paul. 1, 9, 5 } \\
\text { Paul. 1, 9, 5a } \\
\text { Paul. 1, 9, 6 } \\
\text { Paul. 1, 9, 7 } \\
\text { Paul. 1, 9, 8 } \\
\text { Paul. 1, 4, 8 } \\
\text { Interpr. Paul. } \\
\text { sent. 1, 9, 2 } \\
\text { Interpr. Paul. } \\
\text { sent. 1, 9, 3 } \\
\text { Interpr. Paul. } \\
\text { sent. 1, 9, 4 } \\
\text { Interpr. Paul. } \\
\text { sent. 1, 9, 8 } \\
\text { Interpr. Paul. } \\
\text { sent. 1, 9, 1 } \\
\text { Interpr. Paul. } \\
\text { sent. 1, 9, 6 } \\
\text { Interpr. Paul. } \\
\text { sent. 1, 7, 1, } 2 \\
\text { Interpr. Paul. } \\
\text { sent. 1, 9, 5 } \\
\text { Interpr. Paul. } \\
\text { sent. 1, 9, 7 } \\
\text { Interpr. Paul. } \\
\text { sent. 3, 6, 11 } \\
\text { Vat. fr. 151 }\end{array}$ & $\begin{array}{l}\text { Vat. fr. 182 } \\
\text { Vat. fr. 223 } \\
\text { Epit. Ulp. 16, } 1 \\
\text { Epit. Gai. 1, 8, } \\
2 \\
\text { CTh. 2, 9, 3 } \\
\text { Interpr. } \\
\text { CTh. 2, 16, } 2 \\
\text { CTh. 2, 16, 2, } 2 \\
\text { CTh. 2, 16, } 2 \\
\text { Interpr. } \\
\text { CTh. 3, 7, 1 } \\
\text { CTh. 3, 7, 1, } \\
\text { Interpr. } \\
\text { CTh. 3, 32, } 1 \\
\text { CTh. 4, 8, 6, } \\
\text { Interpr. } \\
\text { CTh. 7, 22, } 2 \\
\text { I. 1, 14, 2 } \\
\text { I. 1, 23 } \\
\text { I. 1, 23, 2-3 } \\
\text { I. 2, 19, 5 } \\
\text { I. 2, 19, 6 } \\
\text { I. 4, 6, 33 } \\
\text { D. 1, 7, 17 } \\
\text { D. 2, 8, 8, 1 } \\
\text { D. 2, 8, 8, 2 } \\
\text { D. 2, 15, 9, 3 } \\
\text { D. 3, 3, 51 } \\
\text { D. 4, 1, 8 } \\
\text { D. 4, 3, 7 } \\
\text { D. 4, 3, 38 } \\
\text { D. 4, 4, 1, 1-3 } \\
\text { D. 4, 4 } \\
\text { D. 4, 6 } \\
\text { D. 4, 8, 3 }\end{array}$ & $\begin{array}{l}\text { D. } 4,3,7 \\
\text { D. } 4,4 \\
\text { D. } 4,8,41 \\
\text { D. } 5,1,2,3 \\
\text { D. } 6,1,32 \\
\text { D. } 7,7,6,1 \\
\text { D. } 8,5,15 \\
\text { D. } 9,4,36 \\
\text { D. } 12,2,9,4 \\
\text { D. } 12,6,5 \\
\text { D. } 12,6,67, \\
4 \\
\text { D. } 14,3,11, \\
\text { 1 } \\
\text { D. } 16,1,8, \\
\text { 15 } \\
\text { D. } 16,1,32 \\
\text { D. } 19,1,13, \\
\text { 28 } \\
\text { D. } 20,5,7,1 \\
\text { D. } 21,2,39 \\
\text { D. } 22,6,9 \\
\text { D. } 23,3,6,2 \\
\text { D. } 23,3,62 \\
\text { D. } 26,1,3,1 \\
\text { D. } 26,2,32, \\
2 \\
\text { D. } 26,7,3,3 \\
\text { D. } 26,7,5,6 \\
\text { D. } 26,9 \\
\text { D. } 27,1,10, \\
7 \\
\text { D. } 27,1,44, \\
2 \\
\text { D. } 27,3,20, \\
\text { 1 } \\
\text { D. } 27,6,4 \\
\text { D. } 27,9,1,4 \\
\text { D. } 27,9,11\end{array}$ & $\begin{array}{l}\text { D. } 27,10,16 \\
\text { D. } 28,2,28,3 \\
\text { D. } 28,6,15 \\
\text { D. } 29,2,57 \\
\text { D. } 31,87,1 \\
\text { D. } 32,50,1 \\
\text { D. } 33,1,21,2 \\
\text { D. } 33,1,21,4 \\
\text { D. } 34,1,18,2 \\
\text { D. } 37,10,3,3 \\
\text { D. } 37,14,9,1 \\
\text { D. } 37,14,17 \\
\text { D. } 38,2,8,5 \\
\text { D. } 38,2,14 \\
\text { D. } 38,5,8 \\
\text { D. } 40,2,20 \\
\text { D. } 40,5, \\
\text { 30, } 11 \\
\text { D. } 42,1,2 \\
\text { D. } 42,1,54 \\
\text { D. } 42,1,57 \\
\text { D. } 42,5,5 \\
\text { D. } 44,4,4,26 \\
\text { D. } 48,5,16,6 \\
\text { D. } 49,1,24,1 \\
\text { D. } 49,14,45, \\
\text { 14 } \\
\text { D. } 50,2,6,1 \\
\text { D. } 50,2,11 \\
\text { D. } 50,4,3,10 \\
\text { D. } 50,4,8 \\
\text { D. } 50,5,2 \\
\text { C. } 2,4,36\end{array}$ & $\begin{array}{l}\text { C. } 2,20,7 \\
\text { C. } 2,21,3 \\
\text { C. } 2,21,4 \\
\text { C. } 2,21,5,1 \\
\text { C. } 2,22,2 \\
\text { C. } 2,24,2 \\
\text { C. } 2,25,1 \\
\text { C. } 2,27,1 \\
\text { C. } 2,37,1 \\
\text { C. } 2,39,1 \\
\text { C. } 2,40,1 \\
\text { C. } 2,41,1 \\
\text { C. } 2,42,1 \\
\text { C. } 2,45,1 \\
\text { C. } 2,45,2 \\
\text { C. } 2,46,3 \\
\text { C. } 2,47,1,1 \\
\text { C. } 2,52,1 \\
\text { C. } 2,52,2 \\
\text { C. } 2,52,3 \\
\text { C. } 2,52,4 \\
\text { C. } 2,52,5 \\
\text { C. } 2,52,7 \\
\text { C. } 3,28,8 \\
\text { C. } 3,28,12 \\
\text { C. } 3,37,4 \\
\text { C. } 3,38,8 \\
\text { C. } 4,19,9 \\
\text { C. } 4,26,5 \\
\text { C. } 4,29,4 \\
\text { C. } 4,29,25\end{array}$ & $\begin{array}{l}\text { C. } 4,38,2 \\
\text { C. } 4,44,5 \\
\text { C. } 4,44,13 \\
\text { C. } 4,44,15 \\
\text { C. } 4,51,4 \\
\text { C. } 4,52,5 \\
\text { C. } 5,1,5 \\
\text { C. } 5,3,8 \\
\text { C. } 5,3,17 \\
\text { C. } 5,4,18 \\
\text { C. } 5,4,20 \\
\text { C. } 5,6,6 \\
\text { C. } 5,30,5 \\
\text { C. } 5,31,6 \\
\text { C. } 5,37,14 \\
\text { C. } 5,39,1 \\
\text { C. } 5,59,4 \\
\text { C. } 5,71,15 \\
\text { C. } 5,72,3 \\
\text { C. } 5,74,3 \\
\text { C. } 6,30,10 \\
\text { C. } 6,31,4 \\
\text { C. } 6,31,6 \\
\text { C. } 6,35,6 \\
\text { C. } 7,62,8 \\
\text { C. } 8,13,23 \\
\text { C. } 8,15,4 \\
\text { C. } 8,37,7 \\
\text { C. } 10,50,1 \\
\text { Nov. Iust. } \\
\text { 123, } 13\end{array}$ \\
\hline $\begin{array}{l}26 \\
\text { years }\end{array}$ & Paul. 2, 19, 13 & CTh. $2,16,2$ & $\begin{array}{l}\text { CTh. 2, 16, } \\
2 \text { Interpr. }\end{array}$ & CTh. $3,32,1$ & D. $23,2,66$ & D. $48,5,7$ \\
\hline $\begin{array}{l}28 \\
\text { years }\end{array}$ & $\begin{array}{l}\text { Interpr. Paul. } \\
\text { sent. } 1,9,3\end{array}$ & $\begin{array}{l}\text { Interpr. Paul. } \\
\text { sent. } 1,9,4 .\end{array}$ & $\begin{array}{l}\text { CTh. 2, 16, } \\
2\end{array}$ & & & \\
\hline $\begin{array}{l}29 \\
\text { years }\end{array}$ & CTh. $2,16,2$ & & & & & \\
\hline $\begin{array}{l}30 \\
\text { years }\end{array}$ & $\begin{array}{l}\text { TH. } 11,89-97 \\
\text { TH. } 11,98-107 \\
\text { G. } 1,17 \\
\text { G. } 1,18\end{array}$ & $\begin{array}{l}\text { G. } 1,20 \\
\text { G. } 1,21 \\
\text { G. } 1,29 \\
\text { G. } 1,31\end{array}$ & $\begin{array}{l}\text { G. } 1,32 b \\
\text { G. } 1,35 \\
\text { G. } 1,39\end{array}$ & $\begin{array}{l}\text { G. 2, } 276 \\
\text { Epit. Ulp. 1, } \\
12 \\
\text { Epit. Ulp. 1, } \\
14 .\end{array}$ & $\begin{array}{l}\text { Epit. Ulp. 3, } 3 \\
\text { Epit. Ulp. 3, } 4 \\
\text { CTh. 2, 16, } 2\end{array}$ & $\begin{array}{l}\text { D. } 10,2 \text {, } \\
39,2 \\
\text { D. } 40,4 \text {, } \\
16 \\
\text { C. } 7,15,2\end{array}$ \\
\hline
\end{tabular}




\begin{tabular}{|c|c|c|c|c|c|c|}
\hline $\begin{array}{l}40 \\
\text { years }\end{array}$ & Nov. Mai. 6, 1 & Nov. Mai. 6, 3 & $\begin{array}{l}\text { Nov. Iust. } \\
123,13\end{array}$ & & & \\
\hline $\begin{array}{l}50 \\
\text { years }\end{array}$ & $\begin{array}{l}\text { L. Reg, 1, } 5 \\
\text { Epit. Ulp. 16, } 1\end{array}$ & $\begin{array}{l}\text { Epit. Ulp. 16, } 3 \\
\text { Epit. Ulp. 16, } 4\end{array}$ & $\begin{array}{l}\text { D. } 1,2,2,50 \\
\text { D. } 19,1,21\end{array}$ & $\begin{array}{l}\text { D. } 38,1,35 \\
\text { C. } 5,4,27\end{array}$ & $\begin{array}{l}\text { C. } 6,58,12 \\
\text { C. } 10,32,13\end{array}$ & $\begin{array}{l}\text { Nov. Iust. } \\
6,6\end{array}$ \\
\hline $\begin{array}{l}55 \\
\text { years }\end{array}$ & D. $50,2,2,8$ & D. $50,2,11$ & C. $10,50,3$ & & & \\
\hline $\begin{array}{l}60 \\
\text { years }\end{array}$ & $\begin{array}{l}\text { Epit. Ulp. 16, } 1 \\
\text { Epit. Ulp. 16, } 3\end{array}$ & $\begin{array}{l}\text { Epit. Ulp. 16, } 4 \\
\text { CTh. 16, 2, } 27\end{array}$ & D. $1,7,15,2$ & $\begin{array}{l}\text { D. } 27,1,15 \text {, } \\
11\end{array}$ & C. $1,3,9$ & C. $5,4,27$ \\
\hline $\begin{array}{l}65 \\
\text { years }\end{array}$ & D. $50,5,1,3$ & D. $50,15,3$ & & & & \\
\hline $\begin{array}{l}70 \\
\text { years }\end{array}$ & $\begin{array}{l}\text { I. } 1,25,13 \\
\text { D. } 27,1,2\end{array}$ & $\begin{array}{l}\text { D. } 28,5,57 \\
\text { D. } 50,2,2,8\end{array}$ & $\begin{array}{l}\text { D. } 50,4,3,6 \\
\text { D. } 50,4,3 \text {, } \\
12\end{array}$ & $\begin{array}{l}\text { D. } 50,5,2,1 \\
\text { D. } 50,5,8\end{array}$ & $\begin{array}{l}\text { D. } 50,6,4 \\
\text { C. } 5,67,1\end{array}$ & $\begin{array}{l}\text { C. } 10,32, \\
10 \\
\text { C. } 10,50,3\end{array}$ \\
\hline
\end{tabular}

\section{Age categories in Roman law}

\begin{tabular}{|c|c|c|c|c|c|c|}
\hline $\begin{array}{l}\text { Adulescentia } \\
\text { (Adolescentia, } \\
\text { Aetas } \\
\text { adolescentis) } \\
\text { This category } \\
\text { meant young } \\
\text { people and } \\
\text { included those } \\
\text { under the age of } \\
\text { 25. This term } \\
\text { appeared in the } \\
\text { source material } \\
\text { of all epochs } \\
\text { (from the } \\
\text { classical era } \\
\text { until the } \\
\text { Justinian era). }\end{array}$ & $\begin{array}{l}\text { CTh. 2, 17, } \\
1 \\
\text { CTh. 9, 13, } \\
\text { 1. Interpr. } \\
\text { CTh. 13, 3, } \\
6 \\
\text { CTh. 14, 9, } \\
3,1 \\
\text { Nov. Mai. } 6 \\
\text { I. 1, 23, } \\
\text { I. 1, 24, } 1 \\
\text { I. 2, 20,3 } \\
\text { I. 3, 6, 9 } \\
\text { D. 2, 2, 3, 1 } \\
\text { D. 3, 1, 3, 3 } \\
\text { D. 3, 3, 39, } \\
6 \\
\text { D. 4, 4, 1, 3 } \\
\text { D. 4, 4, 7, 2 } \\
\text { D. 4, 4, 11, } \\
\text { 2 } \\
\text { D. 4, 4, 27 } \\
\text { D. 4, 4, 27, } \\
\text { 1 } \\
\text { D. 4, 4, 32 }\end{array}$ & $\begin{array}{l}\text { D. } 4,4,41 \\
\text { D. } 4,4,43 \\
\text { D. } 4,4,47 \text {, } \\
1 \\
\text { D. } 4,4,49 \\
\text { D. } 6,1,6 \\
\text { D. } 12,3,4 \\
\text { D. } 13,1,13 \\
\text { D. } 13,5,5 \text {, } \\
9 \\
\text { D. } 13,7,16 \\
\text { D. } 15,4,1, \\
9 \\
\text { D. } 17,1,12, \\
11 \\
\text { D. } 26,1,3, \\
1 \\
\text { D. } 26,7,7, \\
11 \\
\text { D. } 26,7,16 \\
\text { D. } 26,7,32, \\
4 \\
\text { D. } 26,7,32, \\
5 \\
\text { D. } 26,7,39 \\
\text { D. } 26,7,39 \text {, } \\
5\end{array}$ & $\begin{array}{l}\text { D. } 26,7,39, \\
15 \\
\text { D. } 26,7,39, \\
18 \\
\text { D. } 26,7,46 \\
\text { D. } 26,7,46, \\
5 \\
\text { D. } 26,8,21 \\
\text { D. } 26,10,3 \text {, } \\
2 \\
\text { D. } 26,10,3, \\
6 \\
\text { D. } 26,10,7 \\
\text { D. } 27,2,3 \\
\text { D. } 27,2,3, \\
5 \\
\text { D. } 27,3,13 \\
\text { D. } 27,3,16, \\
1 \\
\text { D. } 27,3,20, \\
1 \\
\text { D. } 27,4,1, \\
2 \\
\text { D. } 27,6,12 \\
\text { D. } 27,7,7 \\
\text { D. } 27,8,1 \text {, } \\
6 \\
\text { D. } 27,9,3, \\
5\end{array}$ & $\begin{array}{l}\text { D. } 27,9,8 \\
\text { D. } 27,9,8 \text {, } \\
1 \\
\text { D. } 27,9,10 \\
\text { D. } 32,69,1 \\
\text { D. } 35,1,84 \\
\text { D. } 36,1,1 \text {, } \\
12 \\
\text { D. } 36,1,1 \text {, } \\
14 \\
\text { D. } 36,4,5 \text {, } \\
20 \\
\text { D. } 38,10, \\
\text { 10, } 14 \\
\text { D. } 40,4,53 \\
\text { D. } 42,8,10 \text {, } \\
5 \\
\text { D. } 43,24,3 \text {, } \\
3 \\
\text { D. } 45,1,91, \\
3 \\
\text { D. } 46,3,95 \text {, } \\
3 \\
\text { D. } 46,3,96 \text {, } \\
1 \\
\text { D. } 46,6 \\
\text { D. } 46,7,3 \text {, } \\
6\end{array}$ & $\begin{array}{l}\text { D. } 47,2,33 \text {. } \\
\text { D. } 47,2,68, \\
2 \\
\text { D. } 49,4,1 \text {, } \\
13 \\
\text { D. } 49,9,2 \\
\text { D. } 49,14 \text {, } \\
18,8 \\
\text { C. } 1,3,31 \\
\text { C. } 2,12,11 \\
\text { C. } 2,40,2 \\
\text { C. } 2,44,2 \\
\text { C. } 5,31,1 \\
\text { C. } 5,34,12 \\
\text { C. } 5,37,1 \\
\text { C. } 5,37,2 \\
\text { C. } 5,37,5 \\
\text { C. } 5,39,1 \\
\text { C. } 5,41,1 \\
\text { C. } 5,52,2\end{array}$ & $\begin{array}{l}\text { C. } 5,52,2 \text {, } \\
3 \\
\text { C. } 5,61,1 \\
\text { C. } 5,62,2 \\
\text { C. } 5,69,1 \\
\text { C. } 5,69,1 \text {, } \\
1 \\
\text { C. } 5,70,2 \\
\text { C. } 5,71,1 \\
\text { C. } 5,71,4 \\
\text { C. } 7,62,10, \\
1 \\
\text { C. } 9,1,2,1 \\
\text { C. } 11,19,1 \text {, } \\
3 \\
\text { Nov. Iust. } \\
72,2 \\
\text { Nov. } \\
72,3 \\
\text { Nov. } \quad \text { Iust. } \\
72,5 \\
\text { Nov. } \\
72,7 \\
\text { Nov. Iust. } \\
72,8\end{array}$ \\
\hline & CTh. 9, 8, 1 & C. $2,3,22$ & C. $5,62,15$ & C. $9,10,5$ & & \\
\hline
\end{tabular}

The term meaning adult age, which 
occurred only in

classical and

post-classical

imperial

legislation. It

defined persons

who exceeded,

12 (women) and

14 (men) year of

life - i.e.

persons who

were formally

mature.

\section{Anniculus \\ The term \\ referred to a one-year child, which was a category typical for classical law. This category meant the completion of 365 days, since the adoption of the Julian calendar.}

\section{Annositas}

The term used in the postclassical law to describe old age.

\section{Auxilium aetas \\ The term appeared in the sources of classical law and defined people who could benefit from the protection resulting from the restitutio in integrum. The term defined persons up to the age of 25 .}

Firma aetas This category
G. 1,29
G. 1,73
D. $1,6,6$
D. $23,4,26$
D. $28,6,45$
D. 50,16 ,
G. $1,32 \mathrm{a}$ 3
Vat. fr. 321
$5 \quad 10$
D. $9,2,23$,
.
D. 50,16 ,
134

CTh. 1, 15, CTh. 12, 1, CTh. 13, 6, 12113

$\begin{array}{lll}\text { D. } 14,3,11, & \text { D. } 38,9,2 & \text { D. } 10,2,57 .\end{array}$

1
CTh. 2, 16,
CTh. 2, 17,
CTh. 9, 43,
CTh. 16,
C. $1,11,5$
C. $9,51,13$, CTh. 2, 17, CTh. 2, 17, 
determined the

$1,3 \quad 1,4$. Interpr.

stable age and

appeared in the

venia aetatis

institution. It

included men of

20 and women

over 18 years of

age.

\section{Flora aetas}

The term

meaning

blooming age. It

appeared in

post-classical

law to describe

young men who

avoided serving

in the army. He

included men

aged 18-19.

\section{Grandis natu}

The term used to describe the old age, which referred to people around 50 years old.

\section{Imperfecta aetas}

This term described persons up to 25 years of age. It was mentioned only in the Justinian era.

\section{Impubertas}

Age category meaning immature people. The final limits of immature age were 14 years for men and 12 years for women (see pubertas).
CTh. 7, 20, 12

D. $1,2,2, \quad$ D. $50,2,11$ 50

C. $2,40,5$
G. 1, 93

G. 1,102

G. 1,144

G. 1,157

G. 1,165

G. 1, 167

G. 1,179

G. 1,189

G. 2, 179

G. 2,181

G. 2,182

G. 2,183

G. 3, 208

Paul. 2, 19,

1

$\begin{array}{lll}\text { D. } 1,7,22, & \text { D. } 27,1,30, & \text { D. } 29,1,28 \\ 1 & 3 & \text { D. } 29,2,8, \\ \text { D. } 1,7,22, & \text { D. } 27,1,37 & 1 \\ 2 & \text { D. } 27,2,5 & \text { D. } 29,2,11 \\ \text { D. } 1,7,32 & \text { D. } 27,3,4, & \text { D. } 29,2,25 \text {, } \\ \text { D. } 2,4,22 & 2 & 12 \\ \text { D. } 3,5,30, & \text { D. } 27,3,4, & \text { D. } 29,2,40 \\ 5 & 3 & \text { D. } 29,2,42 \\ \text { D. } 3,5,30, & \text { D. } 27,3,9, & \text { D. } 29,2,57 \\ 6 & \text { 1 } & \text { D. } 29,2,57, \\ \text { D. } 4,4,3, & \text { D. } 27,3,18 & 2 \\ 11 & \text { D. } 27,5,1, & \text { D. } 29,2,59 \\ \text { D. } 5,1,12, & 1 & \text { D. } 29,4,27 \\ 2 & \text { D. } 27,5,1, & \text { D. } 29,4,27, \\ \text { D. } 5,2,8,5 & 4 & 1 .\end{array}$

D. $5,2,8,5 \quad 4$
D. $36,1,83$

D. $36,2,7$,

4

D. $36,2,7$

$5 \quad 1$

D. $36,4,5, \quad$ D. $41,2,1$, $12 \quad 13$

D. $37,1,7, \quad$ D. $42,4,3$, 13

$\begin{array}{ll}\text { D. } 37,1,15 & \text { D. } 42,5,23\end{array}$

$\begin{array}{ll}\text { D. } 37,5,5 & \text { D. } 42,5,28\end{array}$

D. $37,5,5, \quad$ D. $42,6,1$, 1 D. 7

D. $37,5,5$, D. $42,8,10$,

11 


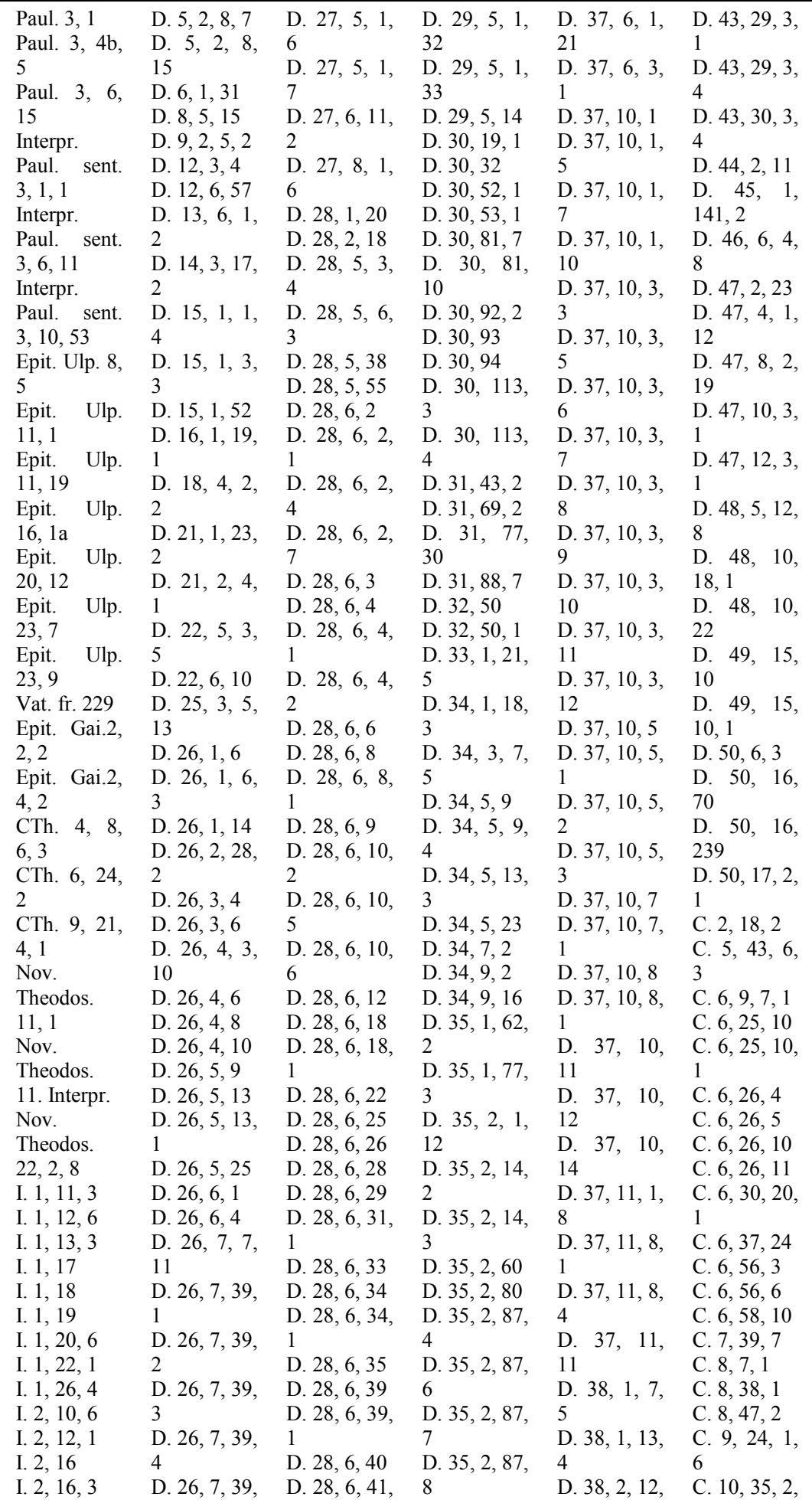




\begin{tabular}{|c|c|c|c|c|c|c|}
\hline $\begin{array}{l}\text { I. } 2,16,4 \\
\text { I. } 2,16,6 \\
\text { I. } 2,16,7 \\
\text { I. } 3,3,6 \\
\text { I. } 3,19,10 \\
\text { I. 4, } 1,18 \\
\text { (20) } \\
\text { D. } 1,6,4 \\
\text { D. } 1,7,20 \\
\text { D. } 1,7,22\end{array}$ & $\begin{array}{l}7 \\
\text { D. } 26,7,39 \text {, } \\
17 \\
\text { D. } 26,7,40 \\
\text { D. } 26,7,58 \\
\text { D. } 26,7,58 \text {, } \\
1 \\
\text { D. } 26,8,13 \\
\text { D. } 26,8,19 \\
\text { D. } 26,10,7 \\
\text { D. } 27,1,28 \text {, } \\
1\end{array}$ & $\begin{array}{l}3 \\
\text { D. } 28,6,41 \text {, } \\
6 \\
\text { D. } 28,6,41 \text {, } \\
7 \\
\text { D. } 28,6,42 \\
\text { D. } 28,6,43 \text {, } \\
3 \\
\text { D. } 28,6,45 \\
\text { D. } 28,6,46 \\
\text { D. } 28,6,47\end{array}$ & $\begin{array}{l}\text { D. } 35,2,95 \text {, } \\
1 \\
\text { D. } 35,3,1 \text {, } \\
1 \\
\text { D. } 35,3,1 \text {, } \\
3 \\
\text { D. } 36,1,11 \text {, } \\
1 \\
\text { D. } 36,1,76 \text {, } \\
1 \\
\text { D. } 36,1,78\end{array}$ & $\begin{array}{l}2 \\
\text { D. } 38,2,16 \text {, } \\
5 \\
\text { D. } 38,2,42, \\
2 \\
\text { D. } 38,5,13 \\
\text { D. } 38,9,1 \text {, } \\
4 \\
\text { D. } 38,9,1 \text {, } \\
13 \\
\text { D. } 38,16,1 \\
\text { D. } 38,17,2 \text {, } \\
5 \\
\text { D. } 38,17,2 \text {, } \\
12 \\
\text { D. } 38,17,2 \text {, } \\
29 \\
\text { D. } 38,17,2 \text {, } \\
30 \\
\text { D. } 38,17,2 \text {, } \\
46 \\
\text { D. } 40,4,22 \\
\text { D. } 40,5,26 \text {, } \\
5 \\
\text { D. } 40,5,41 \text {, } \\
10 \\
\text { D. } 40,5,51, \\
11 \\
\text { D. } 40,7,2, \\
1\end{array}$ & $\begin{array}{l}7 \\
\text { Nov. } \\
22,24 \\
\text { Nov. } \\
22,32 \\
\text { Nov. } \\
22,40 \\
\text { Nov. } \\
72 \\
\text { Nov. } \\
72,2 \\
\text { Nov. } \\
158 \\
\text { Nov. } \\
159 \\
\text { Nov. } \\
159,2\end{array}$ & $\begin{array}{l}\text { Iust. } \\
\text { Iust. } \\
\text { Iust. } \\
\text { Iust. }\end{array}$ \\
\hline
\end{tabular}

\section{Immatura \\ In classical and post-classical sources this term was used as a definition of an immature girl.}

\section{Infantia}

Age category for people in childhood. It covered children under the age of 3 in archaic law. In the postclassical law, the 7-year boundary was used for the first time This limit was definitively adopted by the emperor Justinian. From that time, the category of
D. 24, 1, 32, D. 47, 10, Paul. 1, 13a, 27 25 6

\begin{tabular}{|c|c|c|c|c|c|}
\hline G. 3,109 & I. $2,19,4$ & D. $19,2,19$, & D. $39,1,10$ & D. $45,1,1$ & C. $6,9,3$ \\
\hline Paul. 3, 6, & I. $3,19,10$ & 7 & D. $39,1,11$ & D. $45,1,70$ & C. $6,12,2$ \\
\hline & I. $4,18,4$ & D. $23,4,26$ & D. $40,1,25$ & D. $45,3,20$ & C. $6,30,18$ \\
\hline Paul. 4, 8, 5 & D. $1,5,15$ & D. $25,4,1$, & D. $40,2,24$ & D. $47,2,23$ & C. $6,30,18$, \\
\hline Paul. 5, 4, 2 & D. $1,7,42$ & 15 & D. $40,5,26$, & D. $47,2,68$, & 1 \\
\hline Vat. fr. 89 & D. $2,4,4$ & D. $26,1,3$, & 2 & 2 & C. $6,30,18$, \\
\hline CTh. 4, 1, 1 & D. $3,5,28$ & & D. $40,5,30$, & D. $48,5,12$, & 2 \\
\hline CTh. 4, 1, & D. $4,6,22$, & D. $26,7,2$ & 1 & & C. $6,30,18$, \\
\hline 1. Interpr. & 2 & D. $26,7,2$, & D. $40,5,30$, & D. $48,8,12$ & 3 \\
\hline CTh. 5, 10, & D. $6,1,60$ & & 4 & D. $50,1,21$, & C. $6,56,1$ \\
\hline & D. $6,2,12$, & D. $26,9,7$ & D. $40,5,30$, & & C. $6,61,8$, \\
\hline CTh. 5, 10, & & D. 32,41 , & & D. $50,13,1$, & \\
\hline 1. Interpr. & D. $7,1,12$, & & D. $40,5,30$, & & C. $7,32,3$ \\
\hline CTh. & 3 & D. $33,7,12$, & 6 & D. 50,16 , & C. $7,40,2$ \\
\hline & D. $7,1,55$ & 7 & D. $40,5,30$, & 209 & C. $8,48,5$ \\
\hline CTh. 8, 18, & D. $8,2,5$ & D. $34,1,15$ & 7 & D. 50,16 , & C. $8,51,3$, \\
\hline & D. $9,2,5,2$ & D. $34,2,25$, & D. $40,5,36$ & 246 & 2 \\
\hline CTh. & D. $9,2,23$, & 9 & D. $40,12,6$ & C. $1,4,31$ & C. $8,53,26$, \\
\hline & 7 & D. $36,1,38$, & D. 40,12 , & C. $1,9,7$ & 1 \\
\hline CTh & D. 1 & 1 & 12 , & C. $1,11,7$ & C. $S$ \\
\hline 1. In & D. $13,1,13$ & D. $36,1,67$, & D. $41,2,1$, & C. $3,28,33$, & C. $10,41,1$, \\
\hline
\end{tabular}




\begin{tabular}{|c|c|c|c|c|c|}
\hline $\begin{array}{l}\text { children's age } \\
\text { was set at the } \\
\text { limit of } 7 \text { years. }\end{array}$ & $\begin{array}{l}\text { CTh. 11, } \\
27,1 \\
\text { CTh. 15, } \\
\text { 14, } 7 \\
\text { CTh. 16, 7, } \\
5 \\
\text { I. } 1,23,6\end{array}$ & $\begin{array}{l}3 \\
\text { D. } 37,1,7 \text {, } \\
2 \\
\text { D. } 37,3,1 \\
\text { D. } 37,10,3 \text {, } \\
4 \\
\text { D. } 38,17,2 \text {, } \\
13\end{array}$ & $\begin{array}{l}5 \\
\text { D. } 41,2,32, \\
2 \\
\text { D. } 41,3,28 \\
\text { D. } 44,4,4, \\
9\end{array}$ & $\begin{array}{l}1 \\
\text { C. } 5,10,1 \text {, } \\
1 \\
\text { C. } 5,27,11 \text {, } \\
4\end{array}$ & $\begin{array}{l}1 \\
\text { Nov. Iust. 3, } \\
3\end{array}$ \\
\hline
\end{tabular}

\begin{tabular}{|c|c|c|c|c|c|c|}
\hline $\begin{array}{l}\text { Infantia } \\
\text { proximus }\end{array}$ & $\begin{array}{l}\text { G. } 3,109 \\
\text { I. } 3,19,10\end{array}$ & G. 3,208 & I. $3,19,10$ & $\begin{array}{l}\text { I. 4, } 1,18 \\
(20)\end{array}$ & $\begin{array}{l}\text { D. } 4,3,13 \text {, } \\
1\end{array}$ & $\begin{array}{l}\text { D. 50, } 17 \\
111\end{array}$ \\
\hline
\end{tabular}

Pubertati

proximus

The term

infantia

proximus meant

people close to

childhood.

Sources do not allow to set age limits assigned to this term. It is assumed that the term described people who had already left formal childhood, but were at a similar age. The term pubertati proximus described people who were close to formal maturity but did not reach it yet.

\section{Infirmitas aetatis}

The term found in the classical law denotes the weakness of age. It was used both to determine the inexperience of people under the age of 25 , as well as to underline the physical weakness of a person in old
Paul. 1, 7, $\quad$ CTh. 2, 16, D. 4, 4, 18, Epit. Ulp. 11,1
2, 4. Interpr. 1 CTh. 4, 12, D. 27, 10, 9
D. 30,122

D. $33,1,21$,

D. $46,6,4$, 8

$$
3
$$

2

D. 48,19 ,
C. $5,60,1$ 
age.

Integra aetas

The term

appeared in the post-classical

sources defining people who

could benefit

from the

protection

resulting from

the restitutio in

integrum, i.e. up

to the age of 25 .

\section{Iusta aetas}

The term meant the appropriate age. The jurists themselves were wondering

whether the term should have been used to designate formally mature people, that is, 12 (women) or 14 (men) years old, or it was 25 years old.

\section{Iuventa}

This category included young people. It was assumed that this term was not a technical term of Roman law, despite the fact that it often occurred in the preserved source material. An analysis of classical sources indicates that the term could have been used for 25 years, and in the Justinian law for 25 years.
C. $2,23,1$
C. $6,61,8$,
CTh. 2, 16,
CTh. 4, 22,
2, 4. Interpr.
1. Interpr.
D. $12,4,8$
D. $32,50,6$
D. $50,5,2$

\begin{tabular}{|c|c|c|c|c|c|}
\hline $\begin{array}{l}\text { Vat. fr. } 236 \\
\text { Vat. fr. } 237\end{array}$ & $\begin{array}{l}\text { CTh. 16, } \\
10,19\end{array}$ & $\begin{array}{l}\text { D. } 4,4,25 \text {, } \\
1\end{array}$ & $\begin{array}{l}\text { D. } 40,5,15 \\
\text { D. } 47,21,2\end{array}$ & $\begin{array}{l}\text { D. } 49,1,24 \text {, } \\
1\end{array}$ & $\begin{array}{l}\text { C. } 6,61,8 \text {, } \\
5 \mathrm{a}\end{array}$ \\
\hline $\begin{array}{l}\text { CTh. 2, 17, } \\
1\end{array}$ & $\begin{array}{l}\text { Nov. } \\
\text { Theodos. }\end{array}$ & $\begin{array}{l}\text { D. } 4,4,27 \text {, } \\
1\end{array}$ & $\begin{array}{l}\text { D. } 48,4,1 \text {, } \\
1\end{array}$ & $\begin{array}{l}\text { D. } 49,1,28 \text {, } \\
2\end{array}$ & $\begin{array}{l}\text { C. } 7,25,1 \\
\text { C. } 9,15,1 \text {, }\end{array}$ \\
\hline CTh. 2, 17, & $22,1,3$ & D. $12,1,26$ & D. $48,5,16$, & D. $49,16,4$, & 1 \\
\hline & Nov. Val. 2, & D. $24,3,22$, & 6 & 11 & C. 10 , \\
\hline CTh. 7, 1, & & & D. $48,6,6$ & C. $1,17,2$ & 63 \\
\hline & Nov. Mai. 6 & D. $27,1,6$, & D. 48,13 , & C. $5,50,2$, & Nov. Iust. 5, \\
\hline CTh. 9, 13, & Nov. Mai. & $\begin{array}{lll}5 & & \\
& & \end{array}$ & $\begin{array}{ll}12,1 \\
\end{array}$ & 1 & 3 \\
\hline $\begin{array}{l}\text { CTh. 13, 3, } \\
11 \\
\text { CTh. 16, 2, } \\
10\end{array}$ & $\begin{array}{l}\text { I. } 4,6,33 \\
\text { D. } 2,15,8 \text {, } \\
10\end{array}$ & D. $40,4,52$ & 28,3 & $\begin{array}{l}2 \\
\text { C. } 5,59,4\end{array}$ & $\begin{array}{l}112,3 \\
\text { Nov. Iust. } \\
115,5\end{array}$ \\
\hline
\end{tabular}

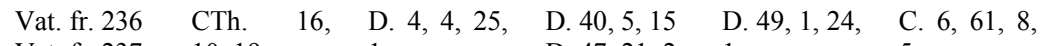
CTh. $2,17, \quad$ Nov.

D. $48,4,1$ $\begin{array}{lll}\text { D. } 49,1,28, & \text { C. } 7,25,1\end{array}$

2

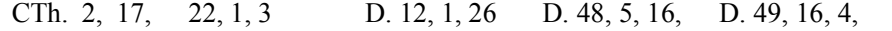

1, 3 Nov. Val. 2, D. 24, 3, 22,

D. $48,6,6$

63

$10 \quad$ Nov. Mai. 6 D. 27, 1, 6,

12,1

3

Cis
11 D. $2,15,8$,
D. $4,4,24$, 2




\begin{tabular}{lllllll}
\hline egitima aetas & Vat. fr. 69 & CTh. 16,2, & D. $46,1,41$ & C. $2,26,1$ & C. $5,42,1$ & C. $7,62,10$, \\
Category & CTh. $2,6,4$ & 11 & D. $49,1,28$, & C. $2,40,2$ & C. $5,46,2$ & 1 \\
indicating the & CTh. 2, 17, & D. $3,5,26$ & 2 & C. $2,44,4$ & C. $5,70,1$ & C. $9,51,13$, \\
legal age & $1,4$. Interpr. & D. $26,2,32$, & C. $2,4,4$ & C. $2,45,1$ & C. $5,73,1$ & 1 \\
(majority). This & CTh. 8,18, & 2 & C. $2,4,5$ & C. $2,52,2$ & C. $6,53,5$ & C. $10,50,2$ \\
category was & 2 & D. $27,7,1$ & C. $2,12,12$, & C. $5,37,12$ & & C. $10,52,6$ \\
introduced in & CTh. 9,43, & D. $34,3,20$, & 1 & & Nov. \\
pre-classical law & CTh. & 1 & C. $2,24,1$ & & 115,3 \\
in 191 or 192 & 17,1 & D. $39,4,16$, & & & &
\end{tabular}

$\mathrm{BC}$ under the lex

(P) laetoria de circumscriptione adulescentium. This age category began after the age of 25.

\section{Maturitas / \\ Matura aetas}

The term

meaning

maturity,

however, there

are no

categorical

sources that

would allow to

determine the

exact age limits.

Nubilis aetas

(Nuptiarum

aetas)

The term

meaning age

suitable for

marriage. In the

Justinian era it

was set that

meant 12 years

for women and

14 for men.

\section{Partus}

The term

defined the

newborn. Due to

the frequency of

appearing in

legal sources,

we can include

it in the

technical terms
CTh. 7, 13, CT $12 \quad 20,3$

CTh. 11, 1, CTh

11, CTh. 16, 5, $29,3 \quad 33$
CTh.

10,10
16

D. $33,2,42$

C. $7,61,2$

\section{$\begin{array}{lll}\text { D. } 35,1,10 & \text { D. } 36,1,11, & \text { C. } 5,4,24\end{array}$}

Paul. 2, 5, $\quad$ D. 5, 3, 20, $\quad$ D. 21, 1, 31,

Paul. 2, 17

Paul. 3, 6, 19

Paul. 4, 9, 5

Paul. 5, 25, $1 \mathrm{~b}$

Epit. Ulp. 3,$$
3
$$

Epit. Ulp. 5 ,

9
D. $5,3,25$,

20

D. $5,3,27$

D. 21, 1, 31, 6

D. $6,1,16$

D. $6,1,20$

D. $21,2,8$

D. $21,2,42$

D. $22,1,4$

D. $6,2,11$,

1

D. $6,2,11$,

D. $22,1,8$

D. $22,1,14$
D. $5,3,20$, D.
D. $25,5,1$

D. $25,6,1$

D. $36,4,5$,

D. $37,9,1$,

D. $26,2,19$,

15

D. $37,10,1$

D. $27,2,1$,

1

11

D. $37,10,3$,

D. $28,2,25$,

1

D. $30,39,1$

D. 30,84 ,
D. $38,8,1, \quad$ C. $6,2,12$ 9

D. $39,5,11$ 2
D. 48,18 17, 2

D. 49,15 , 12, 18

C. $5,13,1$, $9 \mathrm{a}$

C. $5,27,11$, 4

C. $6,35,11$, 
of Roman law. Sources do not allow to set specific age limits of this category.

Epit. Ulp. 5,
10
CTh. $4,8,4$
CTh. 5, 1,
1. Interpr.
Vat. fr. 65
Vat. fr. 114
I. $2,1,37$
I. $2,20,17$
D. $1,5,7$
D. $1,5,14$
D. $1,6,8$
D. $3,5,3,6$
D. $4,2,12$

D. $4,2,12$
D. $7,1,68 \quad$ D. $22,1,14$,

D. $11,8,2 \quad 1$

D. $12,4,12$ D. $22,1,28$,

D. $12,6,15 \quad 1$

D. $12,6,65$, D. $23,3,58$,

5

D. $15,1,57$,

2

$\begin{array}{ll}\text { D. } 18,1,8 & \text { D. } 24,1,28 \text {, }\end{array}$

D. $18,1,31 \quad 5$

D. $18,2,4$, D. $24,3,66$,

1

D. $18,2,4$, D. $25,3,3$, 2

D. $19,1,21$ D. $25,4,1$,

D. $20,1,15$

D. $25,4,1$,

13

D. $25,4,2$,

10

D. $30,91,7$

D. 31,48

D. 31,77 ,

17

D. $33,8,8$,

8

D. $34,9,16$

D. $35,2,9,2$

$1 \quad$ D. $42,5,24$

D. $35,2,24$, D. $42,8,25$,

1

D. $35,2,30$

D. $36,1,23$,

3

D. $36,1,60$,

4

D. $36,2,25$,

5

D. 44, 2, 7, Nov. Val.

3 31. Interpr.

D. $47,2,48$, Nov. Iust.

$5 \quad 39,2$

D. $47,2,48$, Nov. Iust.

D. $47,4,1$,

11

D. 48,10 ,

19,1
C. $8,24,1$

C. $8,51,1$

C. $9,22,10$

C. 11,48 ,

89,8
Parvula aetas

The term used

for boyhood.

\section{Perfecta aetas \\ Category \\ indicating the \\ perfect age \\ (completed age). \\ In classical law, \\ it was \\ tantamount to \\ formal maturity, meaning the achievement of the age of 12 by women and 14 years by men. In Justinian law, this term was used to describe the age beginning after 25 years, which was synonymous with the term legitima aetas defining the legal age.}

Plena aetas The term of the post-classical
CTh. 14, 3, 5

\begin{tabular}{|c|c|c|c|c|c|}
\hline $\begin{array}{l}\text { G. } 1,144 \\
\text { G. } 1,189 \\
\text { G. } 1,190 \\
\text { CTh. 3, 1, } \\
\text { 4. Interpr. }\end{array}$ & $\begin{array}{l}\text { CTh. } 3,30 \text {, } \\
6,1 . \text { Interpr. } \\
\text { I. } 1,19 \\
\text { I. } 1,20,6 \\
\text { D. } 4,4,32\end{array}$ & $\begin{array}{l}\text { D. } 22,3,25 \text {, } \\
1 \\
\text { D. } 26,1,16 \text {, } \\
1 \\
\text { D. } 26,4,4 \\
\text { D. } 26,4,8\end{array}$ & $\begin{array}{l}\text { D. } 27,3,9 \text {, } \\
1 \\
\text { C. } 2,44,4 \\
\text { C. } 3,1,13 \text {, } \\
10 \\
\text { C. } 4,29,22\end{array}$ & $\begin{array}{l}\text { C. } 5,74,3 \\
\text { C. } 6,58,15 \text {, } \\
4 \\
\text { C. } 8,15,7 \\
\text { Nov. Iust. } \\
97,6\end{array}$ & $\begin{array}{l}\text { Nov. } \\
100,2 \\
\text { Nov. } \\
117,1 \\
\text { Nov. } \\
118,5\end{array}$ \\
\hline
\end{tabular}

CTh. 6, 4, C. 6, 61, 8, $17 \quad 1 \mathrm{a}$ 
and Justinian

law, meaning an

adult person,

that is, already

over 25 years of

age.

\section{Plena \\ pubertas}

I. $1,11,4$ D. $1,7,40$,

The term meant

full maturity.

Only in

Justinian law it

was recognized

that it should be combined with the age limit of 18 years. In the literal sense, it determined the difference in age between people.

\section{Praetextata}

aetas

$\begin{array}{lll}\text { D. } 43,30,3, & \text { C. } 4,16,4\end{array}$

This category included people who were of an age who have not yet

authorized them to wear a virilis gown that is a symbol of adulthood. Sources do not define exactly the age of these people. It is assumed that the change of $\operatorname{tog} a$ took place at the age of several years, that is from 14 to 17 or 18 years. Hence, the term praetextata aetas referred to persons of this age.

$\begin{array}{ll}\text { Praetextati } & \text { D. } 43,30,3, \\ \text { proximus } & 6\end{array}$


The term

specifying

persons who are

close to the age

at which the

right to wear a

virilis toga was

acquired, that is,

praetextati

proximus.

Prima aetas /

Secunda aetas

These terms,

appearing in the post-classical and Justinian law, were used to describe the first and second stage of human life. In the simplest terms, they meant immaturity (prima) and formal maturity (secunda), although they could also refer to people like minores and maiores.

\section{Puberes minores et maiores $X X V$ annis}

The term introduced in the pre-classical law under the act (P)laetoria de circumscriptione adulescentium from the second century BC. It meant people under and above the age of 25 .
Nov. Mai. 6 D. $35,2,68$
C. $6,26,10$ C. $6,30,20$, C. $6,26,10,1$
CTh. 3, 17, 3,2 . Interpr
D. $50,4,3$, 15
C. $6,61,8$, $1 \mathrm{~b}$

\begin{tabular}{|c|c|c|c|c|c|}
\hline G. 2,163 & D. $4,1,8$ & D. $22,6,9$ & D. $37,10,3$, & D. $40,5,30$, & C. $3,28,8$, \\
\hline G. 4,57 & D. $4,4,3,2$ & D. $27,6,4$ & & & \\
\hline G. Aug. 26 & D. $4,4,6$ & D. $4,4,30$ & D. $44,1,7$, & D. $23,3,62$ & C. $2,39,1$ \\
\hline Paul. 1, 9, & D. $4,8,3$ & D. $26,7,3$, & 1 & D. 43,24 , & C. $4,38,2$ \\
\hline & D. $4,4,3,6$ & 3 & D. $3,3,51$ & 15,6 & C. $3,28,16$ \\
\hline Paul. 1, 9, & D. $2,8,8,1$ & D. $28,6,2$, & D. $46,8,3$ & D. $29,2,57$, & C. $2,46,3$ \\
\hline & D. $4,4,11$, & & D. $4,4,48$, & & C. $2,47,1$, \\
\hline Paul. 1, 13a, & & D. $4,4,32$ & 2 & D. $42,1,5$ & \\
\hline & D. $4,4,13$, & D. $16,1,8$, & D. $4,4,50$ & D. $42,1,57$ & C. $8,37,7$ \\
\hline Paul. 5, 12, & & & D. $50,2,6$, & D. $46,1,64$ & C. $4,44,5$, \\
\hline & D. $12,6,5$ & D. $4,4,33$ & 1 & D. $31,87,1$ & \\
\hline Paul. 5, 12, & D. $1,7,17$ & D. $27,9,1$, & D. $19,1,13$, & D. 49,14 , & C. $4,29,25$ \\
\hline & D. $14,6,3$, & & & 45,14 & C. $2,52,5$ \\
\hline Interpr. & 2 & D. $4,4,34$ & D. $21,2,39$ & D. $46,3,95$, & C. $4,51,4$ \\
\hline Paul. sent. & D. $12,2,9$, & D. $4,4,36$ & D. $40,2,20$ & 3 & C. $5,59,4$ \\
\hline 1,9 & & D. $42,1,2$ & D. $50,2,11$ & D. $46,3,98$, & C. $5,72,3$ \\
\hline Vat. fr. 182 & D. $4,4,20$, & D. $27,9,11$ & D. $40,5,26$, & 2 & C. $5,71,15$ \\
\hline I. $1,14,2$ & 1 & D. $16,1,32$ & 1 & C. 2,21 & \\
\hline I. $1,23,3$ & D. $14,3,11$, & D. $42,5,5$ & D. $48,5,16$, & C. $2,22,2$ & \\
\hline I. $2,19,6$ & & D. $4,8,41$ & 6 & C. $2,12,14$ & \\
\hline I. $4,6,33$ & D. $26,1,3$, & & D. $44,4,4$, & C. $4,26,5$ & \\
\hline D. 4,6 & D. $4,4,25$ & & & C. $2,37,1$ & \\
\hline D. $5,1,2,3$ & & & D. $44,4,4$, & & \\
\hline
\end{tabular}


26

D. $32,50,3$

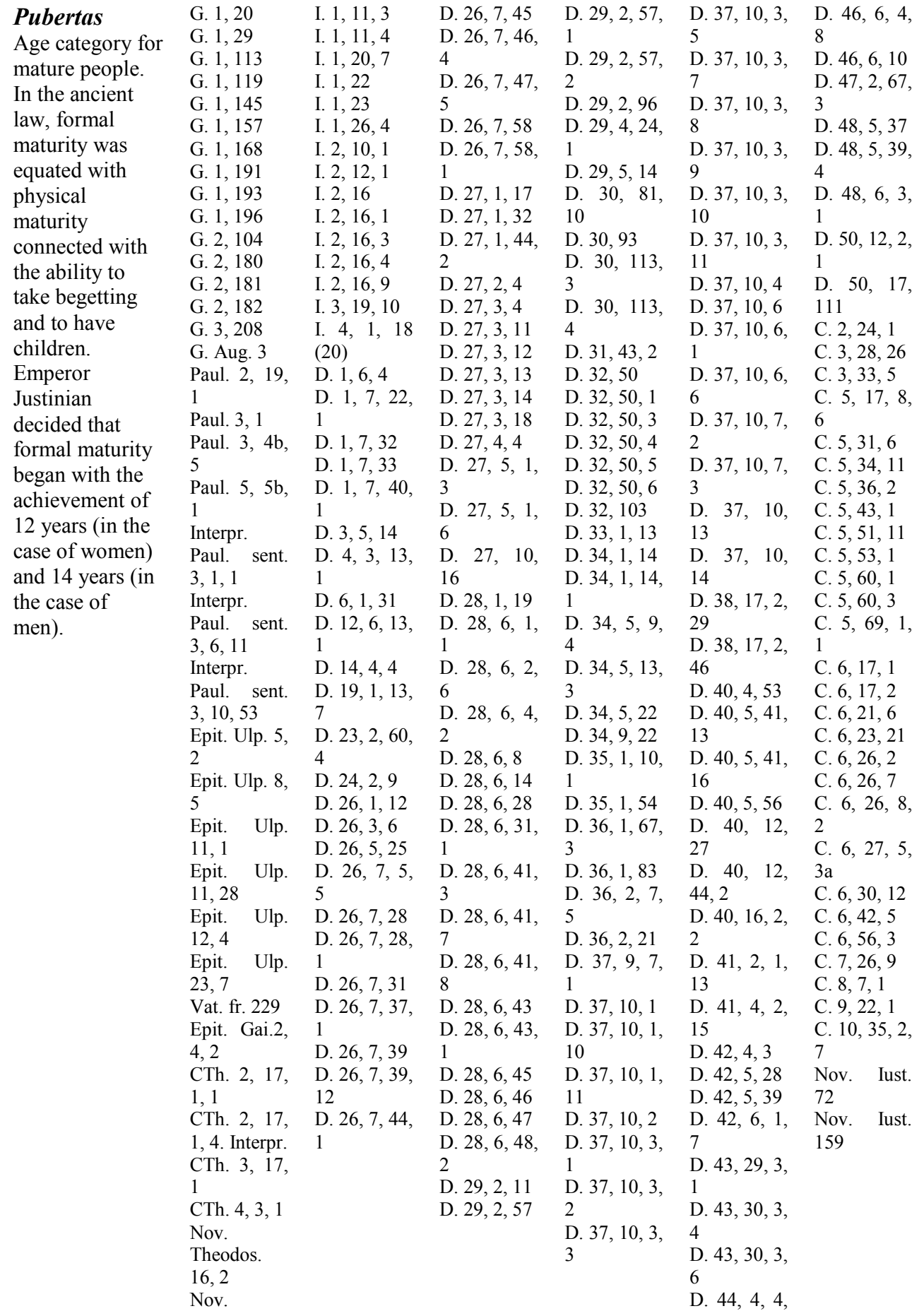


Theodos

22, 2,8

Nov.

Theodos.

22,

Interpr.

I. 1,10
26

D. $44,7,43$

D. 45,1 ,

101

D. 45,1 ,

141,2

D. $46,2,9$

D. $46,2,29$

\section{Pueritia}

Category for

boys' age. As

sources of

classical law

point out, it included people under 17 years

of age.

\section{Pupillaris \\ aetatis \\ (pupillaribus \\ annis)}

The term

pupillus itself

meant a person

subject to

custody, that is,

care and

guardianship.

The term of

pupillaris

aetatis

(pupillaribus

annis) defined

the age category

in a way and

meant people

already formally

mature, but

before reaching

the age of 25 .

\section{Robusta \\ aetatis}

The term created by Justinian compilers, used to determine the oak age (strong, important). It included people over 25 years of age. $\begin{array}{lll}\text { CTh. 2, } 17, & \text { D. } 3,1,1,3 & \text { D. } 37,10,3, \\ 1,3 & & 10\end{array}$

$$
10
$$

C. $2,21,3$
C. $3,6,1$

C. $5,28,3$

C. $5,39,4$

C. $5,59,2$

C. $5,71,16$

C. $6,26,7$

Paul. sent. 1, 5. Interpr. $\quad$ D. 26, 2, 32,

$1,4,2$

CTh. 3, 5, 2

$8 \quad$ Nov. Val. 27

CTh. 3, 18, 35, 13

$1 \quad$ D. $4,4,38$

CTh. 4, 14,

1,2

D. $48,5,16$,

6 


Senecta
This category
meant old age.
Sources of
Roman law do
not specify
when the
beginning of old
age took place.
It is assumed
that it began at
the age of $60-70$
years.

Tenera aetas

The term means delicate age, used in classical and Justinian law. Most probably, the term was used to describe people under the age of 25.

The above presented Index shows the catalogue of all legal Roman law texts where the indications to human's age were mentioned. The most important result of the introduced work proves that in Roman law there was a wide range of age categories and age limits, not only those which we know from the literature i.e. infantia, impubertas and pubertas.

\section{Список використаних джерел}

1. Abdy J. T., Walker B. The Commentaries of Gaius. Cambridge, 1870.

2. Abdy J. T., Walker B. The Institutes of Justinian. Cambridge, 1876.

3. Berger A. Encyclopedic Dictionary of Roman Law. Philadelphia, 1953.

4. Bojarski $W$., Dajczak $W$., Sokala A. Verba iuris, Reguły i kazusy prawa rzymskiego. Torun, 2000.

5. Corpus Iuris Civilis, T. 1 (Institutiones i Digesta) ed. T. Mommsen, P. Krüger; T. 2 (Codex) ed. P. Krüger; T. 3 (Novelle) ed. R. Schöll, G. Kroll, Berlin, 1959-1963.

6. Crawford M. (ed.). Roman Statutes. Vol. 1-2. London, 1996.

7. Dębiński A., Burczak K. Ulpiani Liber singularis regularum. Przekład i objaśnienia. Lublin, 2016.

8. Dębiński A. Zbiór prawa Mojżeszowego i rzymskiego. Tekst łacińsko-polski. Lublin, 2011.

9. Forcellini A. Lexicon Totius Latinitatis. Prati, 1868-1875.

10. Girard P. F., Senn F. Textes de droit romain. Paris, 1890.

11. Heumann H. G. Handlexikon zu den Quellen des römischen Rechts. Graz, 1958.

12. Hornblower S., Spawforth A., Eidinow E. The Oxford Classical Dictionary. Oxford, 2012.

13. Index Interpolationum Quae in Iustiniani Codice Inesse Dicuntur. Tomus in quo ea commemorantur, quae viri docti in scriptis ante annum 1936 editis suspicati sunt / wyd. G. Broggini. Weimar, 1969.

14. Index Interpolationum Quae in Iustiniani Digestis Inesse Dicuntur, Tomus I Ad Libros Digestorum I-XX Pertinens / wyd. E. Levy, E. Rabel. Weimar, 1929.

15. Index Interpolationum Quae in Iustiniani Digestis Inesse Dicuntur, Tomus II Ad Libros Digestorum XXI-XXXV Pertinens / wyd. E. Levy, E. Rabel. Weimar, 1931. 
16. Index Interpolationum Quae in Iustiniani Digestis Inesse Dicuntur, Tomus III Ad Libros Digestorum XXXVI-L Pertinens / wyd. E. Levy, E. Rabel. Weimar, 1935.

17. Kaleta S. Rzymskie prawo prywatne. Wybór źródeł wraz z thumaczeniami. Wrocław, 1954.

18. Koninck de C. Beknopte encyclopedie van het Romeinse recht. Antwerpen, 2000.

19. Koninck de C. Glossarium van Latijnse en Romeinse rechtstermen. Antwerpen, 1997.

20. Korpanty J. Mały słownik łacińsko - polski. Warszawa, 2004.

21. Kosior $W$. Kategorie i granice wieku oraz ich znaczenie w prawie rzymskim. Warszawa, 2018 (Ph.D. thesis).

22. Kruczkiewicz B. (ed.). Słownik łacińsko-polski. Lwów, 1925.

23. Kruger P. Codex Iustinianus. Berolini, 1877.

24. Kunderewicz C. Instytucje Justyniana. Warszawa, 1986.

25. Kunderewicz C., Rezler J. Gaius. Instytucje. Warszawa, 1982.

26. Kuryłowicz M. Słownik łacińskich terminów, zwrotów i sentencji prawniczych. Lublin, 1995.

27. Leverett F. P. A New and Copious Lexicon of the Latin Language. Boston, 1837.

28. Litewski $W$. Słownik encyklopedyczny prawa rzymskiego. Kraków, 1998.

29. Mommsen T. (ed.). Theodosiani libri XVI cum constitutionibus Sirmondianis et leges novellae ad Theodosianum pertinentes. Berlin, 1954.

30. Oxford Latin Dictionary. Oxford, 1968.

31. Palmirski T. (ed.). Digesta Iustiniani. Digesta Justyniańskie. Tekst i przekład. Tom I-VII.2. Kraków, 2012-2016.

32. Pharr C. (ed.). The Theodosian Code and Novels and the Sirmondian Constitutions. A Translation with Commentary, Glossary, and Bibliography, Vol. 1 i 2. Princeton, 1952.

33. Plezia M. (ed.). Słownik łacińsko-polski. Warszawa, 1998. T. 1-5.

34. Riccobono S. (ed.). Fontes iuris Romani anteiustiniani (F.I.R.A.). Firenze, 1940-1943.

35. Romnikiewicz J., Szymoszek E., Żeber I. Prawo rzymskie. Teksty źródłowe do ćwiczeń. Wrocław, 1998.

36. Rozwadowski W. Gai Institutiones. Instytucje Gaiusa. Poznań, 2003.

37. Sitek $B$. Lex coloniae genetivae iuliae seu ursonensis i lex Irnitiana. Ustawy municypalne antycznego Rzymu. Tekst, tłumaczenie i komentarz. Poznań, 2008.

38. Sitek $B$. Tabula Heracleensis (Lex Iulia municipalis). Tekst, thumaczenie, komentarz. Olsztyn, 2006.

39. Smith $W$. Dictionary of Greek and Roman Antiquities. New York, 1878.

40. Sondel J. Słownik łacińsko-polski dla prawników i historyków. Kraków, 2009.

41. Tarwacka A. «Leges regiae». Tekst - thumaczenie - komentarz // Zeszyty Prawnicze. UKSW 4.1/2004.

42. Watson. A. The Digest of Justinian. Philadelphia 1998. Vol. 1-4.

43. Winniczuk L. (ed.), Słownik kultury antycznej. Grecja. Rzym. Warszawa, 1986.

44. Wołodkiewicz W. (ed.). Prawo rzymskie. Słownik encyklopedyczny. Warszawa, 1986.

45. Zabłocka M., Zabłocki J. Ustawa XII Tablic. Tekst. Tłumaczenie. Objaśnienia. Warszawa, 2003.

46. Zedler J. H. Grosses vollständiges Universal-Lexicon aller Wissenschafften und Künste. Halle, Leipzig, 1732.

47. Кофанов Л. Л. (еd.). Дигесты Юстиниана Т. I-VIII. Москва, 2002-2006.

48. Орач С. М., Тищник Б. Й. Римське Приватне Право. Академічний курс. Київ, 2012.

49. Підопригора О. А., Харитонов С. О. Римське Право. Київ, 2003.

\section{References}

1. Abdy, J. T., Walker, B. (1870). The Commentaries of Gaius. Cambridge.

2. Abdy, J. T., Walker, B. (1876). The Institutes of Justinian. Cambridge.

3. Berger, A. (1953). Encyclopedic Dictionary of Roman Law. Philadelphia. 
4. Bojarski, W., Dajczak, W., Sokala, A. (2000). Verba iuris, Reguły i kazusy prawa rzymskiego. Torun.

5. Corpus Iuris Civilis, T. 1 (Institutiones i Digesta) ed. T. Mommsen, P. Krüger; T. 2 (Codex) ed. P. Krüger; T. 3 (Novelle) ed. R. Schöll, G. Kroll, Berlin 1959-1963.

6. Crawford, M. (ed.). (1996). Roman Statutes. Vol. 1-2. London.

7. Dębiński, A., Burczak, K. (2016). Ulpiani Liber singularis regularum. Przektad i objaśnienia. Lublin.

8. Dębiński, A. (2011). Zbiór prawa Mojżeszowego i rzymskiego. Tekst łacińsko-polski. Lublin.

9. Forcellini, A. (1868-1875). Lexicon Totius Latinitatis. Prati.

10. Girard, P. F., Senn, F. (1890). Textes de droit romain. Paris.

11. Heumann, H. G. (1958). Handlexikon zu den Quellen des römischen Rechts. Graz.

12. Hornblower, S., Spawforth, A., Eidinow, E. (2012). The Oxford Classical Dictionary. Oxford.

13. Index Interpolationum Quae in Iustiniani Codice Inesse Dicuntur. Tomus in quo ea commemorantur, quae viri docti in scriptis ante annum 1936 editis suspicati sunt. Weimar: wyd. G. Broggini, 1969.

14. Index Interpolationum Quae in Iustiniani Digestis Inesse Dicuntur, Tomus I Ad Libros Digestorum I-XX Pertinens. Weimar: wyd. E. Levy, E. Rabel, 1929.

15. Index Interpolationum Quae in Iustiniani Digestis Inesse Dicuntur, Tomus II Ad Libros Digestorum XXI-XXXV Pertinens. Weimar: wyd. E. Levy, E. Rabel, 1931.

16. Index Interpolationum Quae in Iustiniani Digestis Inesse Dicuntur, Tomus III Ad Libros Digestorum XXXVI-L Pertinens. Weimar: wyd. E. Levy, E. Rabel, 1935.

17. Kaleta, S. (1954). Rzymskie prawo prywatne. Wybór źródeł wraz z tlumaczeniami. Wrocław.

18. Koninck, de C. (2000). Beknopte encyclopedie van het Romeinse recht. Antwerpen.

19. Koninck, de C. (1997). Glossarium van Latijnse en Romeinse rechtstermen. Antwerpen.

20. Korpanty, J. (2004). Maty stownik tacińsko - polski. Warszawa.

21. Kosior, W. (2018). Kategorie i granice wieku oraz ich znaczenie $w$ prawie rzymskim. Warszawa, (Ph.D. thesis).

22. Kruczkiewicz, B. (ed.). (1925). Stownik łacińsko-polski. Lwów.

23. Kruger, P. (1877). Codex Iustinianus. Berolini.

24. Kunderewicz, C. (1986). Instytucje Justyniana. Warszawa.

25. Kunderewicz, C., Rezler, J. (1982). Gaius. Instytucje. Warszawa.

26. Kuryłowicz, M. (1995). Stownik łacińskich terminów, zwrotów i sentencji prawniczych. Lublin.

27. Leverett, F. P. (1837). A New and Copious Lexicon of the Latin Language. Boston.

28. Litewski, W. (1998). Słownik encyklopedyczny prawa rzymskiego. Kraków.

29. Mommsen, T. (ed.). (1954). Theodosiani libri XVI cum constitutionibus Sirmondianis et leges novellae ad Theodosianum pertinentes. Berlin.

30. Oxford Latin Dictionary. Oxford, 1968.

31. Palmirski, T. (ed.). (2012-2016). Digesta Iustiniani. Digesta Justyniańskie. Tekst i przekład. Tom I-VII.2. Kraków.

32. Pharr, C. (ed.). (1925). The Theodosian Code and Novels and the Sirmondian Constitutions. A Translation with Commentary, Glossary, and Bibliography, Vol. 1 i 2 . Princeton.

33. Plezia, M. (ed.). (1998). Stownik łacińsko-polski, T. 1-5. Warszawa.

34. Riccobono, S. (ed.). (1940-1943). Fontes iuris Romani anteiustiniani (F.I.R.A.). Firenze.

35. Romnikiewicz, J., Szymoszek, E., Żeber, I. (1998). Prawo rzymskie. Teksty źródtowe do ćwiczeń. Wrocław.

36. Rozwadowski, W. (2003). Gai Institutiones. Instytucje Gaiusa. Poznań.

37. Sitek, B. (2008). Lex coloniae genetivae iuliae seu ursonensis i lex Irnitiana. Ustawy municypalne antycznego Rzymu. Tekst, thumaczenie i komentarz. Poznań.

38. Sitek, B. (2006). Tabula Heracleensis (Lex Iulia municipalis). Tekst, thumaczenie, komentarz. Olsztyn. 
39. Smith, W. (1878). Dictionary of Greek and Roman Antiquities. New York.

40. Sondel, J. (2009). Słownik łacińsko - polski dla prawników i historyków. Kraków.

41. Tarwacka, A. (2004). «Leges regiae». Tekst - thumaczenie - komentarz. Zeszyty Prawnicze UKSW 4.1.

42. Watson, A. (1998). The Digest of Justinian, Vol. 1-4. Philadelphia.

43. Winniczuk, L. (ed.). (1986). Stownik kultury antycznej. Grecja. Rzym. Warszawa.

44. Wołodkiewicz, W. (ed.). (1986). Prawo rzymskie. Stownik encyklopedyczny. Warszawa.

45. Zabłocka, M., Zabłocki, J. (2003). Ustawa XII Tablic. Tekst. Tłumaczenie. Objaśnienia. Warszawa.

46. Zedler, J. H. (1732). Grosses vollständiges Universal-Lexicon aller Wissenschafften und Künste. Halle, Leipzig.

47. Kofanov, L. L. (ed.) (2002-2006). Dyhesty Yustynyana T. I-VIII. Moskva.

48. Orach, Ye. M., Tyschnyk, B. J. (2012). Ryms'ke Pryvatne Pravo. Akademichnyj kurs. Kyiv.

49. Pidopryhora O. A., Kharytonov YE. O. (2003) Rymske Pravo. Kyiv.

\title{
ІНДЕКС РИМСЬКИХ ДЖЕРЕЛ ПРАВА, ПОВ'ЯЗАНИХ 3 ВІКОМ
}

\author{
B. Kociop \\ Жешувський університет, \\ вул. Грюнвальдська, 13, Жешув, Польщча, 35-068, \\ e-mail:wojciech.kosior@gmail.com
}

Римське право $є$ основою для всіх європейських правових систем. Дослідження охоплює проблему важливості людського віку в римському праві. У процесі цих досліджень зроблено спробу відповісти на запитання, як вік впливав на правовий статус людини в античному Римі. Результатом проведеної роботи стало створення індексу правових понять, пов'язаних з віком особи. Сам індекс складається з двох частин: перша джерела римського права, у яких були окреслені межі вікових категорій, і друга - вікові категорії та обмеження, що вони накладали у джерелах римського права. У літературі, що стосується предмета дослідження зустрічаємо тільки три такі вікові категорії: infantia, або дитинство від 0-7 років, impubertas - незрілість 7 до 12 і 14 років і pubertas чи підлітковий вік від 12 до 14 років. Однак проведене дослідження показало, що в римському праві були й інші обмеження та вікові категорії, які мали істотне значення. Серед них можемо виділити такі вікові межі: 3 місяці, 6 місяців, 1 рік, 3 роки, 6 років, 7 років, 8 років, 10 років, 11 років, 12 років, 13 років, 14 років, 15 років, 16 років, 17 років, 18 років, 19 років, 20 років, 24 роки, 25 років, 26 років, 28 років, 29 років, 30 років, 35 років, 40 років, 50 років, 55 років, 60 років, 65 років, 70 років.

Встановлено, що у римському праві також містяться такі вікові категорії, які часто зустрічаємо у досліджуваних джерелах: Adolescentia, Adulescentia, Adulta aetas, Aetas adolescentis, Anniculus, Annositas, Auxilium aetas, Firma aetas, Flora aetas, Grandis natu, Imperfecta aetas, Impubertas, Infantia, Infantia proximus, Infirmitas aetatis, Integra aetas, luventa, Legitima aetas, Maturitas, Nascens infans, Nubilis aetas, Nuptiarum aetas, Partus, Parvula aetas, Perfecta aetas, Plena aetas, Plena pubertas, Praetextata aetas, Praetextati proximus, Prima aetas, Puberes maiores XXV annis, Puberes minores XXV annis, Pubertas, Pubertas proximus, Pueritia, Pupillaris aetatis (pupillaribus annis), Recens natus / infans a sanguine, Robusta aetatas, Secunda aetas, Senecta, Tenera aetas.

Результати дослідження мають важливе значення не тільки для римського, але і для сучасного права, тому що вони дають змогу шукати історичну основу для вікових обмежень, які наявні в сучасних правових системах. Сьогодні вікові обмеження мають 
істотне значення для кримінального права (вік, з якого може наставати кримінальна відповідальність), цивільного права (вікові обмеження цивільної дієздатності), шлюбносімейного права (мінімальний вік для вступу в шлюб), конституційного права (граничний вік перебування на посаді) та багатьох інших галузей права.

Ключові слова: Римське право, вік, infantia, impuberes, puberes.

Стаття: надійшла до редакції 10.09.2018 прийнята до друку 01.11.2018 